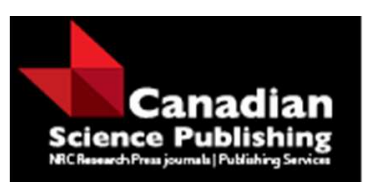

Canadian Journal of Physics

Revue canadienne de physique

\title{
Symmetry breaking and tunneling dynamics of a dipolar Bose-Einstein condensate in a double-well potential
}

\begin{tabular}{|r|l|}
\hline Journal: & Canadian Journal of Physics \\
\hline Manuscript ID & cjp-2017-0565.R1 \\
\hline Danuscript Type: & Article \\
\hline Complete List of Authors: & $\begin{array}{l}\text { Wang, Yuansheng; Guizhou Education University } \\
\text { Long, Ping; Guizhou Education University } \\
\text { Zhang, Bo; Guizhou Education University } \\
\text { Zhang, Hong; Guizhou Education University }\end{array}$ \\
\hline Keyword: & $\begin{array}{l}\text { Bose-Einstein condensate, symmetry breaking, tunneling dynamics, } \\
\text { double-well potential, two-mode model }\end{array}$ \\
\hline $\begin{array}{r}\text { Is the invited manuscript for } \\
\text { consideration in a Special } \\
\text { Issue? : }\end{array}$ & N/A \\
\hline
\end{tabular}




\title{
Symmetry Breaking and Tunneling Dynamics of a Dipolar Bose-Einstein Condensate in a Double-Well Potential
}

\author{
Yuan Sheng Wang,* Ping Long, Bo Zhang, and Hong Zhang \\ School of Physics and Electronic Sciences, \\ Guizhou Education University, Guiyang 550018, China
}

\begin{abstract}
We investigate the properties of a three-dimensional (3D) dipolar Bose-Einstein condensate (BEC) in a double-well potential (DWP). Symmetry breaking and tunneling dynamics phenomena are demonstrated for ${ }^{164}$ Dy atoms in the 3D DWP using an effective two-mode model. The results show that the symmetry properties of the dynamics are affected markedly by the long-range nature and anisotropy of the dipolar interaction and the isotropic contact interaction.

PACS numbers: 03.75.Lm, 34.20.Cf, 32.10.Dk, 32.80.Pj

Keywords: Bose-Einstein condensate, Symmetry breaking, Tunneling dynamics, Double-well potential, Twomode model
\end{abstract}

*Electronic address: joiningnow@126.com; Fax: +86-0851-85811076 


\section{INTRODUCTION}

The Bose-Einstein condensate (BEC) of atoms such as ${ }^{52} \mathrm{Cr},{ }^{164} \mathrm{Dy}$ and ${ }^{168} \mathrm{Er}$ atoms and recent progress on trapping and cooling of polar molecules opens up the study of gases that interact via long-range, anisotropic dipole-dipole interactions (DDIs) [1-10]. In contrast to the isotropic contact interaction present in condensates of alkali atoms, the DDI is long-range and anisotropic, being attractive in certain directions and repulsive in others. As a result, a number of novel phenomena have been predicted to occur in low-density quantum degenerate dipolar atomic system, such as solitons [11,12], droplet formation [13-16], symmetry breaking and vortices in different trapping geometries etc [17-22].

The subject of this paper is to investigate the symmetry breaking and tunneling dynamics of a dipolar BEC in a double-well potential (DWP). Macroscopic dynamical Josephson oscillation and self-trapping in nondipolar BECs have been studied theoretically and partially observed experimentally [23-35]. About spinor dipolar condensates and dipolar condensates Josephson dynamics have also been studied in the literature [17,36-38]. In Ref. 17, Xiong et al. considered a purely dipolar BEC of ${ }^{52} \mathrm{Cr}$ atoms in a DWP and studied the mechanism of the magnetization direction affecting the transition between self-trapping and Josephson oscillation in dynamics. In Ref. 38, Adhikari studied the dynamical self trapping and Josephson oscillation of a repulsive cigar-shaped dipolar ${ }^{52} \mathrm{Cr}$ BEC trapped in an axially-symmetric DWP aligned along the polarization direction using the numerical solution of a mean-field model. The present paper further addresses the Josephson oscillation and self-trapping of ${ }^{164}$ Dy atoms in a DWP system. Compared to ${ }^{52} \mathrm{Cr}$ atom, ${ }^{164}$ Dy has a large magnetic moment $\mu=10 \mu_{B}$ ( $\mu_{B}$ is the Bohr magneton), the dipolar interaction is more strong. It is a good system for us to study the long-range nature and anisotropy of the dipolar interaction affect the symmetry properties of the tunneling dynamics. We derived Josephson type equations in the DWP from Gross-Pitaevskii (GP) equation with the effective two-mode approximations which is proposed by Xiong et al. [17]. The mechanisms of the symmetry properties of the dynamics influenced by the dipolar interaction and the contact interaction are studied in this article, respectively. 


\section{MODEL EQUATIONS}

We consider a dipolar BEC of ${ }^{164}$ Dy atoms confined by a cylindrically symmetric trap in the $x-y$ plane and a double-well structure along the $z$ direction. This kind of geometry is the ideal setup to study the Josephson effect. The trapping potential reads

$$
V(x, y, z)=\frac{m \omega_{\rho}^{2}}{2}\left(\rho^{2}+\lambda^{2} z^{2}\right)+A \exp \left(-z^{2} / 2 \sigma_{0}^{2}\right)
$$

where $\rho^{2}=x^{2}+y^{2}, m$ is the atomic mass, $\lambda=\omega_{z} / \omega_{\rho}$ is the aspect ratio of the trapping potential, $\omega_{\rho}$ and $\omega_{z}$ are the radial and axial angular trap frequencies, respectively. $A$ and $\sigma_{0}$ are the height and the width of a Gaussian profile modeling a barrier between the two potential wells along the $z$ direction. Without loss of generality, we assume that the dipole moments are polarized by the external magnetic field and are constrained to point in the $x-z$ plane. The two-body dipolar interaction potential is then given by

$$
U_{d d}(\mathbf{r})=\mu_{0} \mu^{2}\left[r^{2}-3(z \cos \varphi+x \sin \varphi)^{2}\right] / 4 \pi r^{5},
$$

where $\varphi$ is the angle between the polarized dipole orientation and the $z$ axis, and $\mu_{0}$ is the magnetic permeability of the vacuum. For convenience we define a dimensionless dipolar interaction parameter $D=(N-1) m \mu_{0} \mu^{2} /\left(4 \pi \hbar^{2} a_{h 0}\right)$, where $a_{h 0}=\sqrt{\hbar /\left(m \omega_{z}\right)}$ is the axial harmonic oscillator length and $N$ is the total number of atoms, then a unit system is adopted where the units for length, time, and energy are given by $a_{h 0}, 1 / \omega_{z}$ and $\hbar \omega_{z}$, respectively. Unless specified otherwise, for the numerical results presented below we set $\lambda=1 / 11\left(\omega_{\rho}=2 \pi \times 92.5 \mathrm{~Hz}\right.$ and $\left.\omega_{z}=2 \pi \times 8.4 \mathrm{~Hz}\right), \sigma_{0}=0.2 a_{h 0}$ and $N=200$. In the mean field approximation, a dipolar BEC at sufficiently low temperature is described by a GP equation with nonlocal nonlinearity:

$$
\begin{array}{r}
i \frac{\partial \psi(\mathbf{r}, t)}{\partial t}=\left[-\frac{1}{2} \nabla^{2}+V(\mathbf{r})+4 \pi a N|\psi(\mathbf{r}, t)|^{2}\right. \\
\left.+\int U_{d d}\left(\mathbf{r}-\mathbf{r}^{\prime}\right)\left|\psi\left(\mathbf{r}^{\prime}, t\right)\right|^{2} d^{3} \mathbf{r}^{\prime}\right] \psi(\mathbf{r}, t)
\end{array}
$$

where $\psi(\mathbf{r}, t)$ is the wave function at time $t$ with normalization $\int|\psi(\mathbf{r}, t)|^{2} d \mathbf{r}=1$, $a$ is the atomic scattering length, which can be tuned to a large extent via Feshbach resonance technique. 
In order to investigate the Josephson effect for transitions between the two potential wells, we use two-mode model in the weakly linked limit, which is widely used in the study of BEC and Fermi superfluid in a DWP [17,23-37,39]. We decompose $\psi(\mathbf{r}, t)$ as

$$
\psi(\mathbf{r}, t)=\psi_{1}(t) \Phi_{1}(\mathbf{r})+\psi_{2}(t) \Phi_{2}(\mathbf{r})
$$

where $\psi_{1,2}(t)=\sqrt{N_{1,2}(t)} e^{i \theta_{1,2}(t)}, N_{1,2}$ and $\theta_{1,2}$ are the numbers of atoms and the phases in each of the two wells. The total number of atoms is $N_{T}=\left|\psi_{1}(t)\right|^{2}+\left|\psi_{2}(t)\right|^{2}=N_{1}+N_{2}$. The spatial modes $\Phi_{1,2}(\mathbf{r})$ in Eq.(4) are localized in each of the two wells and are assumed to be real, satisfy the orthonormal condition

$$
\int \Phi_{i}(\mathbf{r}) \Phi_{j}(\mathbf{r}) \approx \delta_{i, j}, i, j=1,2
$$

By inserting Eq.(4) into the GP equation, using the orthogonality and integrating out the spatial degrees of freedom, we obtain the following two-mode equations:

$$
\begin{array}{r}
\dot{\theta}(t)=\Delta E+\Lambda S(t)+\frac{S(t)}{\sqrt{1-S^{2}(t)}} \cos \theta(t), \\
\dot{S}(t)=-\sqrt{1-S^{2}(t)} \sin \theta(t),
\end{array}
$$

where $S(t)=\left[\left|\psi_{1}(t)\right|^{2}-\left|\psi_{2}(t)\right|^{2}\right] / N_{T}$ denotes the population imbalance between the two wells and $\theta(t)=\theta_{2}(t)-\theta_{1}(t)$ is the phase difference. The parameters $\Delta E$ and $\Lambda$ in Eq.(6) are given by

$$
\begin{array}{r}
\Delta E=\frac{E_{1}^{0}-E_{2}^{0}}{2 \kappa}+\frac{\left(U_{1}-U_{2}+A_{11}-A_{22}\right) N_{T}}{4 \kappa}, \\
\Lambda=\frac{\left(U_{1}+U_{2}+A_{11}+A_{22}-2 B_{12}\right) N_{T}}{4 \kappa},
\end{array}
$$

where

$$
\begin{array}{r}
E_{i}^{0}=\int\left[\frac{1}{2}\left|\nabla \Phi_{i}(\mathbf{r})\right|^{2}+V\left|\Phi_{i}(\mathbf{r})\right|^{2}\right] \mathrm{d}^{3} \mathbf{r}, \\
U_{i}=g \int\left|\Phi_{i}(\mathbf{r})\right|^{4} \mathrm{~d}^{3} \mathbf{r}, \\
A_{i i}=\int U_{d d}\left(\mathbf{r}-\mathbf{r}^{\prime}\right)\left|\Phi_{i}(\mathbf{r})\right|^{2}\left|\Phi_{i}\left(\mathbf{r}^{\prime}\right)\right|^{2} \mathrm{~d}^{3} \mathbf{r d}^{3} \mathbf{r}^{\prime}, \\
B_{12}=B_{21}=\int U_{d d}\left(\mathbf{r}-\mathbf{r}^{\prime}\right)\left|\Phi_{1}(\mathbf{r})\right|^{2}\left|\Phi_{2}\left(\mathbf{r}^{\prime}\right)\right|^{2} \mathrm{~d}^{3} \mathbf{r d}^{3} \mathbf{r}^{\prime}, \\
\kappa=-\int\left[\frac{1}{2}\left(\nabla \Phi_{1}(\mathbf{r}) \nabla \Phi_{2}(\mathbf{r})\right)+\Phi_{1}(\mathbf{r}) V(\mathbf{r}) \Phi_{2}(\mathbf{r})\right] \mathrm{d}^{3} \mathbf{r} .
\end{array}
$$


Here we have neglected integrals involving spatial overlaps of $\Phi_{1}(\mathbf{r})$ and $\Phi_{2}(\mathbf{r})$. For two symmetric traps, $E_{10}=E_{20}, U_{1}=U_{2}=U$ and $A_{11}=A_{22}$. The Hamiltonian of the system is

$$
H(S(t), \theta(t))=\frac{\Lambda S^{2}(t)}{2}-\sqrt{1-S^{2}(t)} \cos \theta(t)
$$

Given the initial values $S(0)$ and $\theta(0)$, if $H<-1(\Lambda<0)$ or $H>1(\Lambda>0)$, $S(t)$ will oscillate around a nonzero value. Then we obtain the following two critical values:

$$
\begin{gathered}
\Lambda_{c 1}=\frac{2\left(1+\sqrt{1-S^{2}(0)} \cos \theta(0)\right)}{S^{2}(0)}, \\
\Lambda_{c 2}=\frac{2\left(-1+\sqrt{1-S^{2}(0)} \cos \theta(0)\right)}{S^{2}(0)} .
\end{gathered}
$$

If $\Lambda>\Lambda_{c 1}$ or $\Lambda<\Lambda_{c 2}$, the system is in the self-trapping regime. On the other hand, if $\Lambda_{c 2}<\Lambda<\Lambda_{c 1}$, the system corresponds to the symmetry breaking regime. We use the effective two-mode model which is proposed by Xiong et al. [17]. The basis states $\Phi_{1,2}(\mathbf{r})$ in the two-mode model are used the normalized Gaussian ansatz

$$
\Phi_{i}(\mathbf{r})=\frac{e^{\left[-\left(x+x_{i}\right)^{2} / 2 a_{i}^{2}-\left(y+y_{i}\right)^{2} / 2 b_{i}^{2}-\left(z+z_{i}\right)^{2} / 2 c_{i}^{2}\right]}}{\sqrt{a_{i} b_{i} c_{i}} \pi^{3 / 4}} .
$$

The parameters $a_{i}, b_{i}, c_{i}, x_{i}, y_{i}, z_{i}$, can be obtained by fitting the ground state of the system in our full three-dimensional (3D) simulations of Eq.(3) [18]. For a given value of $\varphi$, the value of $\kappa$ can be obtained analytically, and the values of $A_{11}, B_{12}, U$ and $\Lambda$ can be obtained numerically.

\section{NUMERICAL RESULTS}

The fixed point categories of the system are $z= \pm \sqrt{1-\frac{1}{\Lambda^{2}}}, \theta(0)=0$ for $\Lambda<-1$ in the self-trapping regime and $z=0, \theta(0)=0, \pi$ for $\Lambda_{c 2}<\Lambda<\Lambda_{c 1}$ in the symmetry breaking regime. In order to know the symmetry properties of the dynamics better, we chose initial values $S(0)=0.9, \theta(0)=0$. First, we consider a purely dipolar BEC of ${ }^{164}$ Dy atoms in the DWP with scattering length $a=0(U=0)$ and $A=9 \hbar \omega_{z}$. we study the effect of dipolar strength in the symmetry breaking and tunneling dynamics. Fig. 1(a) gives the values of $A_{11}, B_{12}$ and $\kappa$ vary with the dipole orientation parameter $\varphi$. Fig. 1(b) depicts the values of the parameter $\Lambda$ change with $\varphi$. The system is symmetrical between the two critical values 
$\Lambda_{c 1}$ and $\Lambda_{c 2}$. The value of $\Lambda$ gradually increases and then settles to almost steady value with the increase of $\varphi$. The critical angle from the asymmetric state to the symmetric one is $37.5^{\circ}$, which matches with our previous numerical simulations of Eq.(3), where we observed $\varphi=38^{\circ}$. It can make sure the results of our calculation are reliable. Using the parameters showed in Fig. 1, we study the tunneling dynamics of BEC in the DWP. Fig. 2 shows the averaged population $\langle S\rangle$ with initial values $S(0)=0.9, \theta(0)=0$. With increasing the value of $\varphi$, the system changes from the self-trapping regime into the Josephson-oscillation regime at a critical angle $37.5^{\circ}$. The critical angle is in agreement with the result showed in Fig. 1(b). In order to further understand the effect degrees produced by the dipole orientation on the tunneling dynamics between the two potential wells, the dynamics from the self-trapping regime to the Josephson-oscillation regime is illustrated in Fig. 3(a)-(d). The effective dipolar interaction is attractive for $\varphi=0^{\circ}$, the system is corresponding to the self-trapping state (see inset in Fig. 3(a)). For critical angle $\varphi=37.5^{\circ}$, the system is in the broken-symmetry state by the enhanced repulsion. From $\varphi=40^{\circ}$ to $\varphi=90^{\circ}$, the system is in the Josephson-oscillation regime and the oscillation frequency is gradually raised with the increased repulsive force. The numerical solution of the dynamics for $\varphi=37.5^{\circ}$ is also studied. An initial state with the desired initial population imbalance $S(0)$ is obtained by using Eq.(4). In the numerical simulation, we set $N_{1}=0.05, N_{2}=0.95$. With this initial state we perform the real-time propagation of the 3D GP equation (3) maintaining all the parameters are agreement with the imaginary-time propagation[18]. The results for self-trapping and oscillatory dynamics as obtained from the numerical simulation and the two-mode model are compared in Fig. 3(e). Compare to the two-mode model, the numerical result has higher oscillation frequency and self-trapping degree.

Next we study the effect of the contact strength in the symmetry breaking and tunneling dynamics for a fixed dipole orientation $\varphi=0^{\circ}$ and barrier height $A=9 \hbar \omega_{z}$. Fig. 4(a) gives the values of $A_{11}, B_{12}, \kappa$ and $U$ vary with the scattering length $a$. For $a>0$, the interactions between atoms are repulsive. $U$ rises smoothly with the increase of the scattering length. Fig. 4(b) depicts the values of the parameter $\Lambda$ change with $a$. The system is symmetrical between the two critical values $\Lambda_{c 1}$ and $\Lambda_{c 2}$. The value of $\Lambda$ gradually increases and then settles to almost steady value with the increase of $a$. The critical angle from the asymmetric state to the symmetric one is $a=60 a_{0}$, which matches with our previous numerical simulations of Eq. (3). Using the parameters showed in Fig. 4, we study the tunneling dynamics of BEC in 

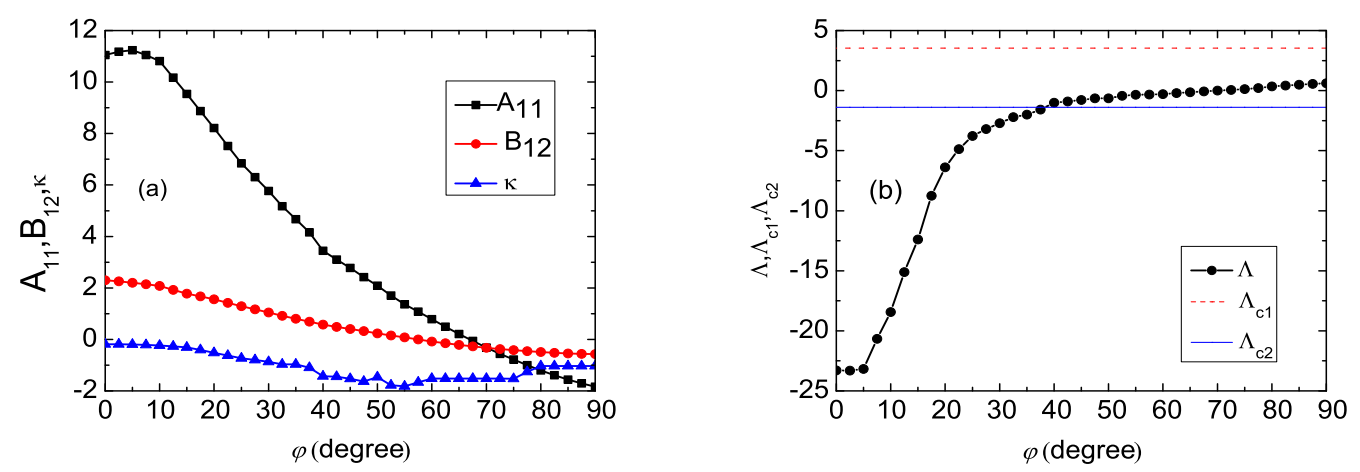

FIG. 1: (Color online) (Color online) (a) Values for the two-mode model parameters $A_{11}, B_{12}$ and $\kappa$ as functions of the dipole orientation parameter $\varphi$. (b) Value of $\Lambda$ as function of the dipole orientation parameter $\varphi . \Lambda_{c 1}=3.54$ and $\Lambda_{c 2}=-1.39$ are the critical values with initial values $S(0)=0.9, \theta(0)=0$.

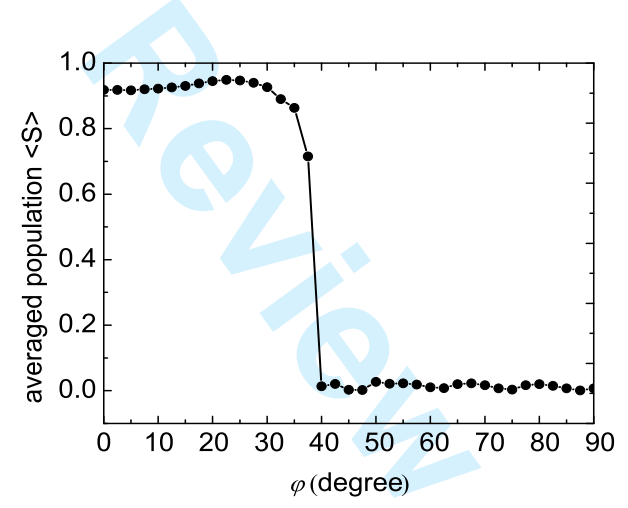

FIG. 2: Averaged population $\langle S\rangle$ as a function of the dipole orientation parameter $\varphi$ with initial values $S(0)=0.9, \theta(0)=0$.

the DWP. Fig. 5 shows the averaged population $\langle S\rangle$ with initial values $S(0)=0.9, \theta(0)=0$. With increasing the value of $a$, the system changes from the self-trapping regime into the Josephson-oscillation regime at a critical value $60 a_{0}$. The critical value is in agreement with the result showed in Fig. 4(b). In order to further understand the effect degrees produced by the contact strength on the tunneling dynamics between the two potential wells, the dynamics from the self-trapping regime to the Josephson-oscillation regime is illustrated in Fig. 6. The dipolar attractive interaction plays more dominant role for $\varphi=0^{\circ}$ and $a=5 a_{0}$ than the contact interaction. The system is corresponding to the self-trapping state (see inset in Fig. 6(a)). For critical value $a=60 a_{0}$, the system is in the broken-symmetry state 

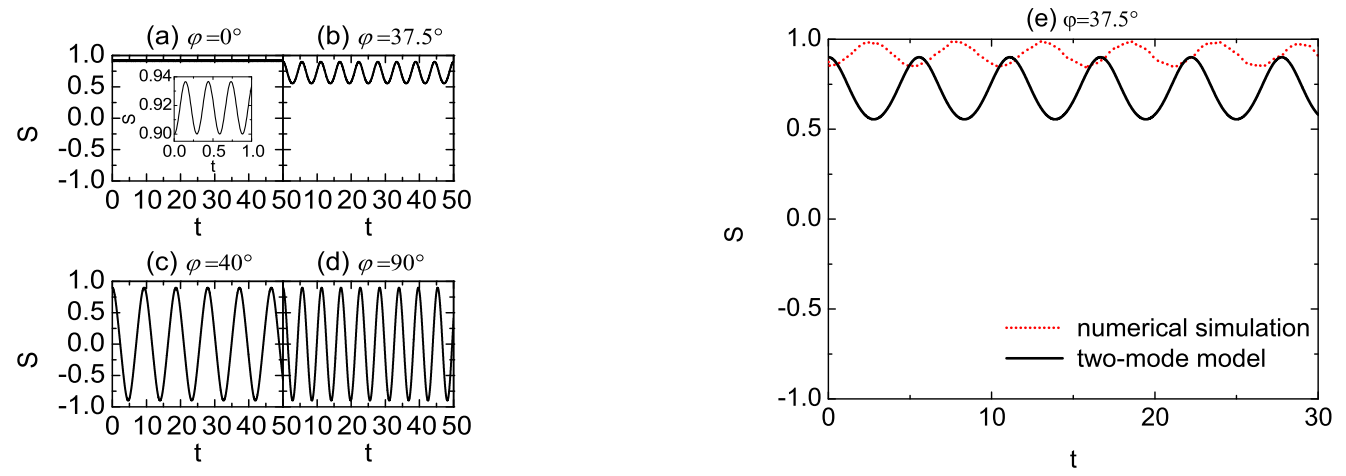

FIG. 3: Population imbalance $S(t)$ versus time for different values of the dipole orientation parameter with initial values $S(0)=0.9, \theta(0)=0$. (a) The system is in the self-trapping regime at $\varphi=0^{\circ}$. (b) The system is in the symmetry breaking regime at critical angle $\varphi=37.5^{\circ}$. (c) The system is in the Josephson-oscillation regime at $\varphi=40^{\circ}$. (d) The system is in the Josephson-oscillation regime at $\varphi=90^{\circ}$ and the oscillation frequency is more quickly as the increased repulsive force. (e) The results for self-trapping and oscillatory dynamics as obtained from the numerical simulation and the two-mode model for $\varphi=37.5^{\circ}$.

by the enhanced repulsion. From $a=65 a_{0}$ to $a=100 a_{0}$, the contact interactions gradually play a dominant role. The contact interactions gradually play a dominant role. The system is in the Josephson-oscillation regime and the oscillation frequency is gradually raised with the increased repulsive force.
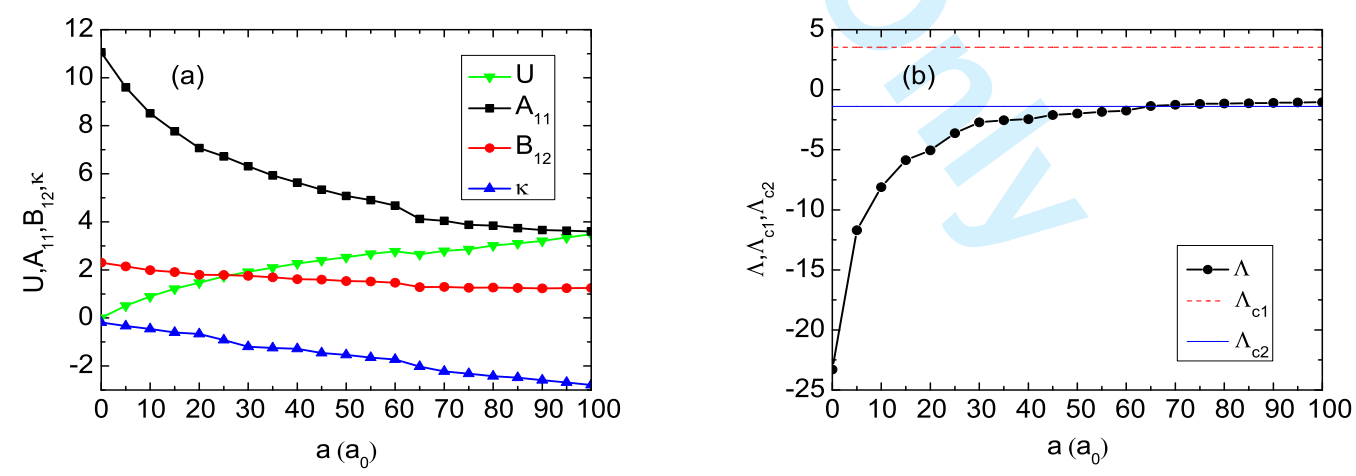

FIG. 4: (Color online) (a) Values for the two-mode model parameters $A_{11}, B_{12}, \kappa$ and $U$ as functions of the scattering length $a$. (b) Value of $\Lambda$ as function of the scattering length $a . \Lambda_{c 1}=3.54$ and $\Lambda_{c 2}=-1.39$ are the critical values with initial values $S(0)=0.9, \theta(0)=0$. 


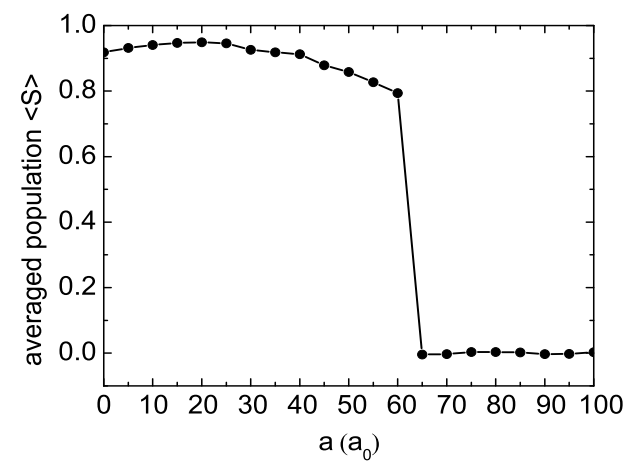

FIG. 5: Averaged population $\langle S\rangle$ as a function of the scattering length $a$ with initial values $S(0)=$ $0.9, \theta(0)=0$.

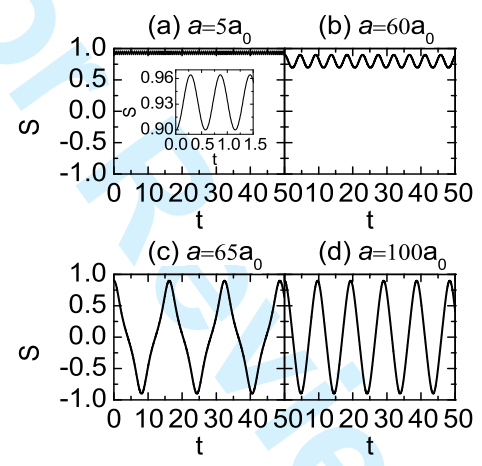

FIG. 6: Population imbalance $S(t)$ versus time for different values of the scattering length with initial values $S(0)=0.9, \theta(0)=0$. (a) The system is in the self-trapping regime at $a=5 a_{0}$. (b) The system is in the symmetry breaking regime at critical angle $a=60 a_{0}$. (c) The system is in the Josephson-oscillation regime at $a=65 a_{0}$. (d) The system is in the Josephson-oscillation regime at $a=100 a_{0}$ and the oscillation frequency is more quickly as the increased repulsive force.

\section{CONCLUSIONS}

In summary, using the effective two-mode mode, we have investigated the symmetry breaking and tunneling dynamics of ${ }^{164}$ Dy atoms in a DWP system. Using the two-mode model basis states obtained by fitting the ground state of the GP equation, we calculated the two-mode equation parameters. Based on these parameters, we studied the symmetry breaking and tunneling dynamics in two main aspects. First, we consider a purely dipolar BEC, we find that the long-range nature and anisotropy of the dipolar interaction affect 
the symmetry properties of the tunneling dynamics. Second, we studied the mechanism of the symmetry properties of the dynamics influenced by the contact interaction for a fixed dipolar strength. Because the increase in the dipole orientation parameter or the scattering length is essentially an increase in repulsive force, so the similar conclusions can be obtained in both ways.

\section{Acknowledgments}

We wish to thank J. Wang for her support. This work was supported by the Science and Technology Foundation of Guizhou Province, China (Grant No. J[2013]2255), the Natural Science Foundation of Guizhou Education University, China (Grant No. 2015BS006) and the National Natural Science Foundation of China (Grant Nos. 11665009 and 11647114).

[1] A. Griesmaier, J. Werner, S. Hensler, J. Stuhler, and T. Pfau, Phys. Rev. Lett. 94, 160401 (2005).

[2] A. Griesmaier, J. Stuhler, and T. Pfau, Appl. Phys. B 82, 211 (2006).

[3] T. Lahaye, T. Koch, B. Fröhlich, M. Fattori, J. Metz, A. Griesmaier, S. Giovanazzi, and T. Pfau, Nature 448, 672 (2007).

[4] T. Koch, T. Lahaye, J. Metz, B. Fröhlich, A. Griesmaier, and T. Pfau, Nat. Phys. 4, 218 (2008).

[5] T. Lahaye, C. Menotti, L. Santos, M. Lewenstein, and T. Pfau, Rep. Prog. Phys. 72, 126401 (2009).

[6] M. Lu, N. Q. Burdick, S. H. Youn, and B. L. Lev, Phys. Rev. Lett. 107, 190401 (2011).

[7] K. Aikawa, A. Frisch, M. Mark, S. Baier, A. Rietzler, R. Grimm, and F. Ferlaino, Phys. Rev. Lett. 108, 210401 (2012).

[8] K. -K. Ni, S. Ospelkaus, M. H. G. de Miranda, A. Pe'er, B. Neyenhuis, J. J. Zirbel, S. Kotochigova, P. S. Julienne, D. S. Jin, and J. Ye, Science 322, 231 (2008).

[9] M. H. G. de Miranda, A. Chotia, B. Neyenhuis, D. Wang, G. Quéméner, S. Ospelkaus, J. L. Bohn, J. Ye, and D. S. Jin, Nat. Phys. 7, 502 (2011).

[10] A. Chotia, B. Neyenhuis, S. A. Moses, B. Yan, J. P. Covey, M. Foss-Feig, A. M. Rey, D. S. 
Jin, and J. Ye, Phys. Rev. Lett. 108, 080405 (2012).

[11] M. J. Edmonds, T. Bland, and D. H. J. O’Dell, Phys. Rev. A 93, 063617 (2016).

[12] X. Jiang, Z. Fan, Z. Chen, W. Pang, Y. Li, and B. A. Malomed, Phys. Rev. A 93, 023633 (2016).

[13] I. Ferrier-Barbut, H. Kadau, M. Schmitt, M. Wenzel, and T. Pfau, Phys. Rev. Lett. 116, 215301 (2016).

[14] K. T. Xi and H. Saito, Phys. Rev. A 93, 011604 (2016).

[15] F. Wächtler and L. Santos, Phys. Rev. A 93, 061603 (2016).

[16] C. Mishra and R. Nath, Phys. Rev. A 94, 033633 (2016).

[17] B. Xiong, J. Gong, H. Pu, W. Bao, and B. Li, Phys. Rev. A 79, 013626 (2009).

[18] Y. S. Wang, Z. Y. Li, Z. W. Zhou, and X. F. Diao, Phys. Lett. A 378, 48 (2014).

[19] Y. S. Wang, Z. W. Zhou, Z. Y. Li, and G. F. Liu, J. Phys. Soc. Jpn. 83, 063002 (2014).

[20] Y. S. Wang, S. T. Ji, Y. E. Luo, and Z. Y. Li, J. Korean Phys. Soc. 67, L1504 (2015).

[21] T. Li, S. Yi, and Y. Zhang, Phys. Rev. A 93, 053602 (2016).

[22] R. K. Kumar, T. Sriraman, H. Fabrelli, P. Muruganandam, and A. Gammal, J. Phys. B: At. Mol. Opt. Phys. 49, 155301 (2016).

[23] A. Smerzi, S. Fantoni, S. Giovanazzi, and S. R. Shenoy, Phys. Rev. Lett. 79, 4950 (1997).

[24] S. Raghavan, A. Smerzi, S. Fantoni, and S. R. Shenoy, Phys. Rev. A 59, 620 (1999).

[25] S. K. Adhikari, Eur. Phys. J. D 25, 161 (2003).

[26] L. Morales-Molina and J. B. Gong, Phys. Rev. A 78, 041403(R) (2008).

[27] E. A. Ostrovskaya, Y. S. Kivshar, M. Lisak, B. Hall, F. Cattani, and D. Anderson, Phys. Rev. A 61, 031601(R) (2000).

[28] A. Trombettoni and A. Smerzi, Phys. Rev. Lett. 86, 2353 (2001).

[29] J. Javanainen, Phys. Rev. Lett. 57, 3164 (1986).

[30] J. E. Williams, Phys. Rev. A 64, 013610 (2001).

[31] S. Giovanazzi, A. Smerzi, and S. Fantoni, Phys. Rev. Lett. 84, 4521 (2000).

[32] I. Zapata, F. Sols, and A. J. Leggett, Phys. Rev. A 57, R28 (1998).

[33] M. Albiez, R. Gati, J. Fölling, S. Hunsmann, M. Cristiani, and M. K. Oberthaler, Phys. Rev. Lett. 95, 010402 (2005).

[34] R. Gati and M. K. Oberthaler, J. Phys. B: At. Mol. Opt. Phys. 40, R61 (2007).

[35] F. S. Cataliotti, S. Burger, C. Fort, P. Maddaloni, F. Minardi, A. Trombettoni, A. Smerzi, 
and M. Inguscio, Science 293, 843 (2001).

[36] M. Yasunaga and M. Tsubota, Phys. Rev. A 81, 023624 (2010).

[37] M. Abad, M. Guilleumas, R. Mayol, M. Pi, and D. M. Jezek, Europhys. Lett. 94, 10004 (2011).

[38] S. K. Adhikari, Phys. Rev. A 89, 043609 (2014).

[39] S. K. Adhikari, H. Lu, and H. Pu, Phys. Rev. A 80, 063607 (2009). 